the development of novel hybrid derivatives of 1,2,4-triazolethiol hybrids as a inhibitor of HIV and HCV infection.

Method The target compounds were developed via nucleophilic reaction with basic amines/thiols. These molecules have been subsequently tested for anti-HIV activity using TZM-bl cell lines along with Luciferase expression profile of the TZM-bl cells after infecting with NL4.3 virus and MTT assay for the cytotoxicity determination. The anti-HCV activity was also determined by capability to obstruct the HCV replicase (HCV NS5B) activity in vitro and HCV replication in a cell culture system carrying replicating $\mathrm{HCV}$ subgenomic RNA replicon. The metabolic profiling of the active compound was also carried out to define the formation of metabolites by the oral route.

Results Among the tested derivatives, compound 6d found to exhibit $98 \%$ of inhibition of HIV with $\mathrm{Ki}$ of $245.12 \mathrm{nM}$ against HIV-RT with more than 50\% inhibition of HIV replication. Moreover, compound 6e showed significant inhibition of RNA dependent RNA polymerase (RdRp) activity of HCV replicase in vitro with IC507.8 $\mu \mathrm{g} / \mathrm{ml}$. It also showed significant inhibition of $\mathrm{HCV}$ replication in culture system which leads to reduction in HCV RNA titre and translation level of viral proteins in concentration-dependent manner. In metabolic analysis, it has been found that, the skeleton of the hybrid compound remained intact during metabolic assay for the longer duration to exert its effect.

Conclusion Together with excellent bioactivities against HIV and HCV infection and favourable metabolic profile, the derivatives of triazole-thiol showed promising option to develop newer therapeutics against the HIV and HCV coinfection.

\section{P5.04 ACADEMIC DETAILING IN HIV: AN ALTERNATIVE IN PRIMARY CARE}

Ana Amélia Nascimento da Silva Bones, Airton Tetelbom Stein, Silvio César Cazella. Universidade Federal de Ciências da Saúde de Porto Alegre, Brazil

\subsection{6/sextrans-2017-053264.620}

Introdution The 90-90-90 target predicts that by $2030 \mathrm{HIV}$ infection will no longer be a global epidemic. In this context, the Protocol for the Treatment of HIV patients in Primary Care provides that all HIV patients receive the offer of antiretroviral treatment. This challenge requires a process of health education. Academic Detailing (DA) may be an alternative to accelerate the implementation of this new guideline. The purpose of this study is to discuss the principles of $\mathrm{AD}$ review the evidence of its effectiveness in, and potential for improving, HIV care.

Methods This is an ongoing study, with the partial results of the author of the thesis in the doctorate degree program in Health Science promoted by UFCSPA. The method is literature integrative review in which were followed: identification of the problem, defining the objective and lesson plan, searching for literature, the evaluation and analysis of found resources. The general educational research literature on improving physician performance was reviewed along with studies that were designed to test $\mathrm{AD}$.

Results The study show that AD could modify doctors' practices in primary care, when you understand the reasons for the behaviour. Brief reinforcement visits increase success rates and targeting programs to physicians at greatest need improves the cost effectiveness of educational interventions. This educational intervention has already been widely investigated for chronic diseases such as hypertension, diabetes, gynaecological and obstetric diseases. Studies have been conducted by Harvard to implement the treatment of HIV exposure prophylaxis. However, the management of people living with HIV/AIDS was not found in the literature.

Conclusions $\mathrm{AD}$ is one of the few educational interventions that has consistently demonstrated improved medical performance. $\mathrm{AD}$ methods to improve HIV practices in primary care are in need of much additional research. Improving the detection of sexual infections disorders and underuse of HIV health treatment may prove to be more difficult when teamwork don't know the Guidelines.

\section{P5.05 NON-TRADITIONAL STRATEGIES TO APPROACH TOPICS RELATED TO SEXUALLY TRANSMITTED DISEASE}

Ana Maria Viana Pinto, Robson dos Santos Souza Marinho, Maria Clara Rodrigues Vieira. Universidade Federal Fluminense, Rio de Janeiro, Brazil

\subsection{6/sextrans-2017-053264.621}

Introduction The traditional teaching strategies follow steps of activities and demonstrations generally directed by the teacher. This procedure may not provide students with valuable skills or even with knowledge that lasts much beyond the end of the term. To enhance the quality of teaching and learning complex problems in the classroom we use non-traditional strategies to approach topics related to sexually transmitted disease important to the health of groups, communities and general populations. Based in this proposition, professors of the health area need to search new strategies in order to adjust the professional education of the graduates to the health system in order to ensure the delivery of quality healthcare to the population. The purpose of this work was to elaborate hornbook and a board game with specific guiding principles, rules to discuss about the theme sexually transmitted diseases by undergraduate students. Materials and Methods.

Development of didactic materials: the first step was to make literature review about the theme following of the elaboration of hornbook, questions and answers for better understanding and comprehension of the subject. The hornbook and the board game emphasised principles of health education, the conceptual definition, conceptual context of the problem, etiologic agents, method of transmission, mechanisms of prevention, control and therapies.

Dynamic with hornbook For the application of the hornbook people or students were sitting on a circle to discuss about the theme before the board game application.

Dynamic of board game: The classes were divided into groups of five students and received a board, dice, game playing pieces, cards specific with questions and answers, curiosity cards, and instructions.

Results and conclusion games and hornbook therefore, seemed to be valuable tools, not only for students learning, but also for teachers, groups, communities, homeless and general populations. Board games and hornbook can promoting pleasure and could be used to mobilise the desire to know, essential to the learning process at any age application. 